\title{
Influência das Mudanças Climáticas na Aptidão Agrícola para Cultivo de Trigo na Microrregião de Guarapuava, Sul do Brasil
}

\author{
Lauro Augusto Ribas Teixeira $^{1}$ (D), Sidnei Osmar Jadoski ${ }^{1}$, Robert Faggian ${ }^{2}$, Victor Sposito ${ }^{2}$ \\ ${ }^{1}$ Departamento de Agronomia, Universidade Estadual do Centro Oeste, Guarapuava, PR, Brasil. \\ ${ }^{2}$ Centre for Regional and Rural Futures, Deakin University, Melbourne, Australia.
}

Recebido em: 22 de Abril de 2019 - Aceito em: 8 de Abril de 2020

\begin{abstract}
Resumo
As evidências de que ocorrerão mudanças climáticas globais em função do aumento da concentração de gases de efeito estufa têm se apresentado cada vez mais consistentemente e aceitas pela comunidade científica internacional. O objetivo deste trabalho foi avaliar estimativas de alterações climáticas na microrregião de Guarapuava no estado do Paraná, analisando os impactos sobre a cultura do trigo, utilizando modelagem para cenários futuros e comparando com um cenário base do passado. Foram ajustados modelos de adequação matemática utilizando Análise de Múltiplos Critérios (MCA) e Processo de Hierarquia Analítica (AHP), com a finalidade de avaliar os possíveis impactos das mudanças climáticas na cultura de trigo, utilizando os cenários climáticos RCP4.5 e RCP8.5 (IPCC) para o cálculo do índice de aptidão agrícola da cultura na microrregião de Guarapuava-PR. Os resultados indicam que o fator de maior impacto para a redução do índice de aptidão agrícola do trigo nos próximos 30 anos, é a projeção de aumento da temperatura média mensal em aproximadamente 1,5 a $2{ }^{\circ} \mathrm{C}$ durante o ciclo de desenvolvimento da cultura no campo.
\end{abstract}

Palavras-chave: cenários climáticos, análise multicriterial, índice de aptidão agrícola, IPCC.

\section{Influence of Climatic Changes on Agricultural Land Suitability Potential for Wheat Cultivation in the Guarapuava Microregion, Southern Brazil}

\begin{abstract}
Evidence that global climate change will occur as a result of increased concentrations of greenhouse gases has been increasingly consistent and accepted by the international scientific community. This study aimed to evaluate climate change estimates in the Guarapuava microregion in the state of Paraná, analyzing the impacts on the wheat crop, using modeling for future scenarios and comparing with a base scenario of the past. Mathematical adequacy models were adjusted using Multiple Criteria Analysis (MCA) and Analytical Hierarchy Process (AHP), with the purpose of evaluating the possible impacts of climate changes on wheat crop, using the climatic scenarios RCP4.5 and RCP8.5 (IPCC) for the calculation of the Land suitability potential in the Guarapuava-PR microregion. The results indicate that the factor of greatest impact for the reduction of the agricultural aptitude index of the wheat in the next 30 years is the projection of increase of the average monthly temperature by approximately 1.5 to $2{ }^{\circ} \mathrm{C}$ during the development cycle of the crop in the field.
\end{abstract}

Keywords: climatic scenarios, multi-criterial analysis, land suitability potential, IPCC.

\section{Introdução}

As evidências de que ocorrerão mudanças climáticas globais, em função do aumento da concentração de gases de efeito estufa têm se apresentado cada vez mais consistentes, impactando diversas atividades, como a agri- cultura (Gunda, et al., 2017), Biomas, processos energéticos e evidenciando necessidade de ampliar rapidamente os conhecimentos na área (Chao e Feng, 2018). Alley et al. (2007) mostram, inclusive, que mudanças climáticas globais bruscas já ocorreram no passado e podem acontecer

Autor de correspondência: Lauro Augusto Ribas Teixeira, lauroribas@uol.com.br. 
novamente, adiantando os cenários previstos para um futuro mais distante. Segundo O’Neill (2014), há evidências de que o clima do planeta está mudando e que essas mudanças de temperatura, precipitação (quantidade e frequência), $\mathrm{CO}_{2}$ e $\mathrm{O}_{3}$ afetarão paulatinamente a agricultura.

Conforme Trenberth (2011) as tendências evidentes de mudanças de clima e aquecimento global, resultarão em mudanças de padrões com aumento da precipitação global em resposta ao aumento da evaporação das superfícies terrestre e oceânica, mais quentes. Chao e Feng (2018) enfatizam que são necessários sobre mudanças climáticas a fim de avaliar os impactos e desenvolver políticas adequadas. Quanto aos impactos econômicos nas atividades produtivas, Burke et al. (2015) apontam que é importante avaliações com foco local e regional, pois as perdas globais esperadas são aproximadamente lineares na temperatura média global, com perdas medianas muitas vezes maiores do que os principais modelos indicam.

Conforme discutido em EBI et al. (2014), foi desenvolvida uma nova estrutura integrada para facilitar a produção de cenários integrados com base em combinações de projeções do modelo climático, condições socioeconômicas e premissas sobre políticas climáticas. De acordo com O'Neill et al. (2014), um dos principais objetivos desses cenários integrados é facilitar a pesquisa e a avaliação caracterizando o grau de incerteza nos esforços de mitigação necessários para adaptação que poderiam ser empreendidos para se preparar e responder as mudanças climáticas e impactos associados a esses processos.

A comunidade internacional de modelagem climática adotou quatro cenários de alterações de clima através do Painel Intergovernamental sobre Mudanças Climáticas (IPCC), estes cenários no relatório do IPCC de 2013, são chamados de Representative Concentration Pathways (RCPs) (Farag et al., 2016).

Os cenários variam de RCP8.5, que corresponde a um cenário de "política não climática" traduzindo-se em impactos severos da mudança climática, ao RCP2.6. Que representa um sistema de política climática rigorosa para limitar as emissões de gases de efeito estufa, traduzindo-se em impactos de baixa severidade, além de dois cenários intermediários, RCPs 4.5 e 6.0, selecionados para serem espaçados uniformemente entre os RCPs 2.6 e 8.5 (Van Vuuren et al., 2011).

Modelos de cenários climáticos utilizam métodos quantitativos e são desenvolvidos através de uma abordagem estratégica em vários níveis temporais e espaciais (Giorgi, 2005). A Análise de Múltiplos Critérios (MCA) é uma metodologia comumente utilizada para desenvolver tais modelos, utilizando a aptidão agrícola como proxy para os rendimentos de culturas que determinam os valores de todos os atributos ambientais subjacentes (Romeijn et al., 2014). Considerando três variáveis principais: Solo, Topografia e Clima, com pesos atribuídos dependendo de sua influência sobre o crescimento e rendimento das culturas (Sposito e Faggian, 2013).

O trigo (Triticum aestivum L.) é o cereal mais importante para a alimentação humana, é cultura agrícola mais cultivada no mundo, com produção estimada em 730 milhões de toneladas (Michel e Scuze, 2018). O estado do Paraná é o maior produtor de trigo do país, sendo que sua produção, além de ser utilizada na indústria moageira local, também atende uma parcela significativa da demanda da Região Sudeste.

Cereais de inverno como o trigo apresentam desenvolvimento vegetativo e reprodutivo altamente dependentes da temperatura média do ar durante o ciclo de cultivo (Silva et al., 2011). Streck e Alberto (2006) destacam possíveis efeitos negativos do aumento futuro da temperatura do ar sobre a produtividade do trigo no Sul do Brasil. Para Santi et al. (2017) nos próximos 50 anos o período de cultivo do trigo indicado pelo zoneamento agroclimático será reduzido em toda a região Sul do País, com a temperatura sendo a principal responsável pela limitação do número de decêndios aptos à semeadura. Fallahpour et al. (2012) destacam que a aptidão das terras para determinados cultivos poderá ser prejudicada pelas alterações climáticas em curso.

O objetivo deste trabalho foi avaliar estimativas de alterações climáticas na microrregião de Guarapuava no estado do Paraná, analisando os impactos sobre a cultura do trigo a partir de modelagem para cenários futuros e comparações com um cenário base do passado.

\section{Material e Métodos}

A área de estudo abrangeu a totalidade da Microrregião de Guarapuava que ocupa 1.448.604 hectares do estado do Paraná (Fig. 1).

Geograficamente esta área é composta por 18 municípios: Campina do Simão, Candói, Cantagalo, Espigão

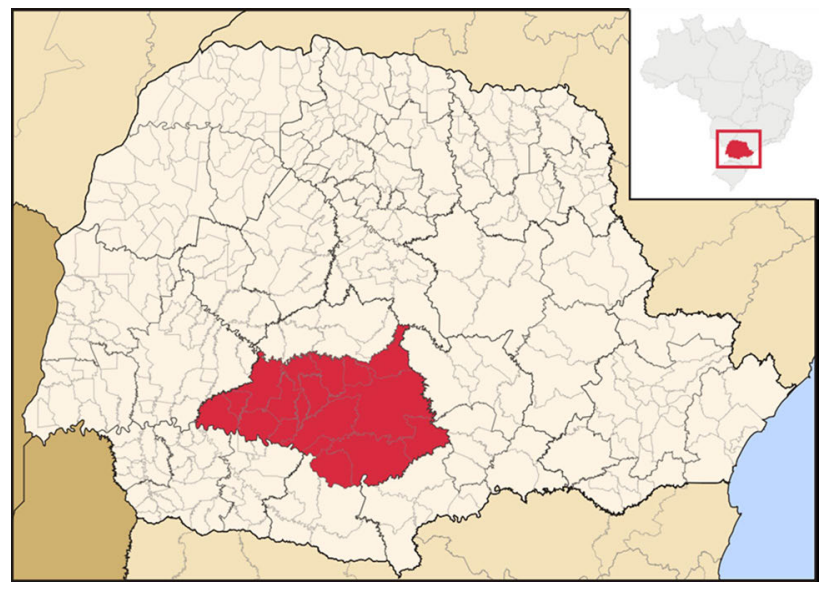

Figura 1 - Microrregião de Guarapuava no estado do Paraná. Fonte: IBGE (2014). 
Alto do Iguaçu, Foz do Jordão, Goioxim, Guarapuava, Inácio Martins, Laranjeiras do Sul, Marquinho, Nova Laranjeiras, Pinhão, Porto Barreiro, Quedas do Iguaçu, Reserva do Iguaçu, Rio Bonito do Iguaçu, Turvo e Virmond, representando aproximadamente $8,1 \%$ do território paranaense. Localizada no Terceiro Planalto Paranaense ou Planalto Basáltico, é a mais extensa das unidades de relevo do estado. Sendo formado por rochas ígneas eruptivas, principalmente basaltos, cuja alteração formou a "popularmente denominada terra vermelha", a partir da Era Mesozoica. O bioma predominante é a floresta subtropical, com vastas áreas de mata de araucárias. Conforme censo agro do IBGE (2017), a microrregião de Guarapuava, abrange um total de aproximadamente 25.000 estabelecimentos rurais em que cerca de $80 \%$ destinam área ao cultivo para culturas anuais. Conforme SEAB (2018), o cultivo de inverno na região é realizado com a as culturas do trigo, cevada e pastagens, sendo ocupados aproximadamente 65 mil hectares com trigo, porém esta área é variável anualmente devido a questões de balança comercial do produto.

Neste estudo foi utilizado o Sistema de Informações Geográficas (SIG) ArcGIS versão 10.3 para processamento de dados e elaboração de mapas de estudo ambiental associado a mudanças climáticas a partir do Modelo Ambiental Global Hadley versão 2 (HadGEM2-ES - Hadley Global Environment Model 2 - Earth System), conforme descrito por Collins et al (2011). Ese modelo foi projetado com o propósito específico de simular e entender a evolução da escala climática, incluindo retornos biogeoquímicos.

Na região de Guarapuava, Teixeira et al. (2018) verificaram alta correlação entre os dados de temperatura gerados pelo modelo HadGEM2-ES, e dados observados de temperatura local. Além disso, para a Bacia do Paraná, que engloba a área de estudo, Benezath et al. (2019) verificaram a adequação do modelo para estimativa de precipitação pluvial, resultados que demostram adequação do modelo para estimativas climáticas na região de Guarapuava-PR. Os resultados indicam que o modelo HadGEM2-ES instalado no Instituto Nacional de Pesquisas Espaciais (INPE) fornece resultados satisfatórios em comparação com o seu original no UK Met Office. Além disso, o modelo HadGEM2-ES consegue simular de modo bastante satisfatório o clima sobre a América do Sul, apresentando alguns erros como uma tendência a subestimar a temperatura à superfície, mas reproduzindo bem padrões de precipitação em regiões como a zona de convergência do Atlântico Sul (ZCAS) (Baxter et al., 2008).

Foram avaliados três cenários climáticos, sendo um cenário base, a partir de série temporal de dados climáticos do período 1970-2000 representando o clima normal local, e dois cenários futuros com projeções modeladas para o ano de 2050 como centro do período 2040-2060. Estas projeções foram elaboradas utilizando os cenários RCP4.5 e RCP8.5, considerados de média e alta severidade de alterações climáticas, respectivamente (Van Vuuren et al., (2011).

As varáveis climáticas empregadas no modelo e suas respectivas unidades são médias mensais de Temperatura $\left({ }^{\circ} \mathrm{C}\right)$, precipitação $(\mathrm{mm})$, temperatura máxima $\left({ }^{\circ} \mathrm{C}\right)$, temperatura mínima $\left({ }^{\circ} \mathrm{C}\right)$, radiação solar $\left(\mathrm{W} / \mathrm{m}^{2}\right)$ e umidade relativa $(\%)$.

Tais dados de clima foram obtidos do sistema associado ao modelo HadGEM2-ES do UK Met Office, em que o banco de dados climáticos globais se baseia na interpolação e controle de qualidade dos dados provenientes de aproximadamente 30.000 estações meteorológicas de superfície, além de outras fontes de dados como registros históricos, radar, satélites e modelos numéricos, conforme descrevem Chen et al. (2008) e Martin et al. (2011). Tais dados podem ser obtidos no WorldClim (2020) em: http://www.worldclim.org/ e CGIAR-CSI (2020). - Consortium for Spatial Information em: http:// www.cgiar-csi.org/.

Os dados de variáveis climáticas foram inseridos no banco de dados SIG, adotando o sistema GCS_WGS-84 de coordenadas geográficas, com resolução espacial de 30 " de arco $(0,0110833$ graus decimais $)$ proporcionando uma resolução aproximada de $1 \mathrm{~km}^{2}$ recomendável para aplicações regionais (Sposito e Faggian, 2013). Segundo IBGE (2006), na Resolução IBGE ${ }^{\circ}$ 23, de 21 de fevereiro de 1989, o WGS84 e o SIRGAS2000 podem ser considerados atualmente coincidentes.

As superfícies têm uma resolução espacial de 30 segundos de arco; isso é equivalente a cerca de $0,86 \mathrm{~km}^{2}$ no Equador e comumente referido como resolução espacial de "1 km". Os dados são referidos como "WorldClim versão 2" e estão disponíveis para download, no formato grid, em http://worldclim.org/ (HIJMANS et al., 2005).

Para estimar o índice de aptidão agrícola (IAP) da cultura do trigo foi utilizado o método Análise de Múltiplos Critérios (MCA) conforme descrito por Sposito e Faggian (2013), onde os atributos com seus componentes são ponderados e analisados utilizando o Processo de Hierarquia Analítica (AHP) desenvolvido por Saaty (1987). Neste processo são utilizados atributos ambientais considerando 3 variáveis principais: Solo, Topografia e Clima.

Para a variável solo foram utilizados dados provenientes da plataforma ISRIC - The World Data Centre for Soils (Batjes, 1995; hengl et al., 2014). Foram utilizados dados de relevo e topografia provenientes da Empresa Brasileira de Pesquisa Agropecuária, disponíveis para download em Embrapa (2017).

No caso da variável "solo" seu valor na estrutura final para o cálculo do índice de aptidão agrícola foi ponderado de acordo com método descrito por Mrazova et al. (2017). Os atributos utilizados foram os seguintes: $\mathrm{pH}$ $\left(\mathrm{CaCl}_{2}\right)$ para as camadas da superfície do solo $(0-0,10 \mathrm{~m}) \mathrm{e}$ 
camada de 0,10-0,30 m; Capacidade de troca de cátions (CTC), Matéria orgânica (M.O.) e Textura para a camada de 0-0,30 m além de Profundidade de Rocha e Profundidade efetiva. Para a variável Topografia foram considerados os atributos declive, vertente e altitude. A variável Clima foi dividida em dois atributos principais: temperatura e precipitação. Posteriormente esses atributos foram ponderados de acordo com as caraterísticas fenológicas da cultura (Cunha et al., 2015).

Essas variáveis e seus atributos foram valorados, sendo as faixas de valores definidas para atender ao rendimento ótimo, com índices que variam de 0,0 a 1,0, dependendo do Índice de aptidão agrícola de 0\% a 100\% para análise no processo AHP. Os valores médios fixados para a variação dos atributos foram ajustados considerando-se características eco fisiológicas, necessidades hídricas e térmicas da cultura sendo ponderados com informações agronômicas verificadas na literatura (Bassoi et al., 2016; Mohanty et al., 2012; Fernandes et al., 2011). O resultado final para a MCA utilizando AHP, considerando todas as variáveis envolvidas para as culturas de trigo podem ser visualizados na Fig. 2.

Os atributos de cada variável foram ponderados com pesos atribuídos dependendo de sua influência sobre o desenvolvimento e produtividade da cultura do trigo (Fig. 2). A definição do IAP final é matematicamente calculada, a partir das características locais, em que solo e topografia variam pontualmente, pré-definindo um potencial aptidão para a cultura. Neste processo, as variáveis climáticas irão consolidar um determinado nível de IAP, de acordo com as variações apresentadas dentro do cenário base, e daquelas estimadas por modelagem para o futuro, nos diferentes cenários.

As informações referentes as análises do IAP foram então incorporadas em ambiente SIG, produzindo uma representação espacial dos resultados com utilização de Grids. O cálculo final para cada cenário proposto foi realizado utilizando a ferramenta de sobreposição ponderada (Weighted Overlay), levando em consideração todos os Grids, atributos e seus respectivos valores (Ferretti e Pomarico, 2013; Malczewski, 2004; Dujmovic et al., 2009, Bathrellos e Gaki-Papanastassiou, 2013).

Para avaliação dos 03 cenários considerados na área de estudo, foram estabelecidos índices relativos para as variações de temperatura e precipitação pluvial em relação ao cenário base. Estes foram determinados considerando a proporção entre a área abrangida para dados intervalos de temperatura e precipitação, em relação à área total da microrregião. Representando um índice percentual de aumento ou redução das variáveis nos cenários futuros em relação ao cenário base. Para a temperatura foi apresentado o intervalo de confiança em relação à média. Os

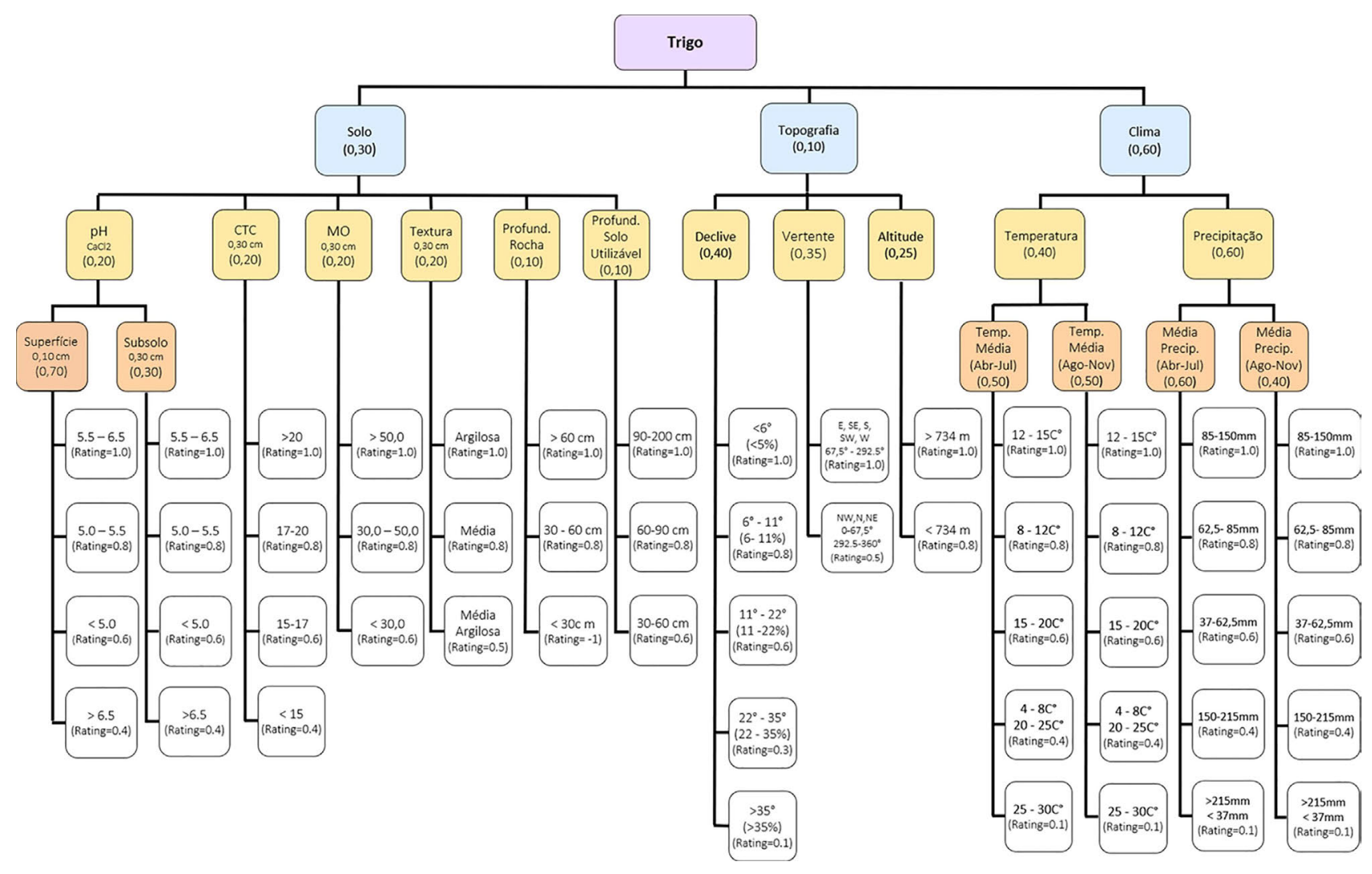

Figura 2 - Análise de Múltiplos Critérios aplicada a cultura de trigo. 
intervalos de temperatura foram definidos a cada $2{ }^{\circ} \mathrm{C}$ entre 10 e $26^{\circ} \mathrm{C}$, e os de precipitação a cada $30 \mathrm{~mm}$ entre 60 e $270 \mathrm{~mm}$, abrangendo os limites históricos e estimados destas variáveis para o local. Para comparação utilizou-se correlação linear de Pearson classificando a significância do coeficiente (r) conforme descrito em Pimentel-Gomes (1990).

\section{Resultados e Discussão}

Os resultados das avaliações do índice de aptidão agrícola para a cultura do trigo referentes ao cenário base (1970 - 2000) apresentados na Fig. 3, demonstram que a maior parte da área de estudo apresentava IAP de $80 \%$. Em alguns setores a oeste de Guarapuava e Inácio Martins é possível observar de maneira mais concentrada áreas com IAP de até 90\%, enquanto que ao sudoeste de Pinhão e a sudeste de Reserva do Iguaçu encontram-se alguns pontos com índice de $70 \%$. Pontos similares a este também aparecem de forma mais dispersa em alguns municípios distribuídos no setor oeste como Espigão Alto do Iguaçu e entornos.

Os valores médios do IAP estimados em porcentagem e suas respectivas distribuições em hectares na área de estudo na projeção climática 2041-2060, considerando os cenários RCP4.5 e RCP8.5 podem ser observados na Tabela 1 e ilustrados nas Figs. 4 e 5. A avaliação dos resultados obtidos para o cenário base (1970-2000) e comparação com cenários futuros demonstra mudança significativa no IAP da área para o cultivo de trigo.

A partir das estimativas inerentes ao cenário RCP4.5 observa-se um aumento de $3 \%$ de áreas com $60 \%$ de IAP ao sul da área de estudo. Áreas localizadas a leste, que anteriormente indicavam um índice de aptidão agrícola de

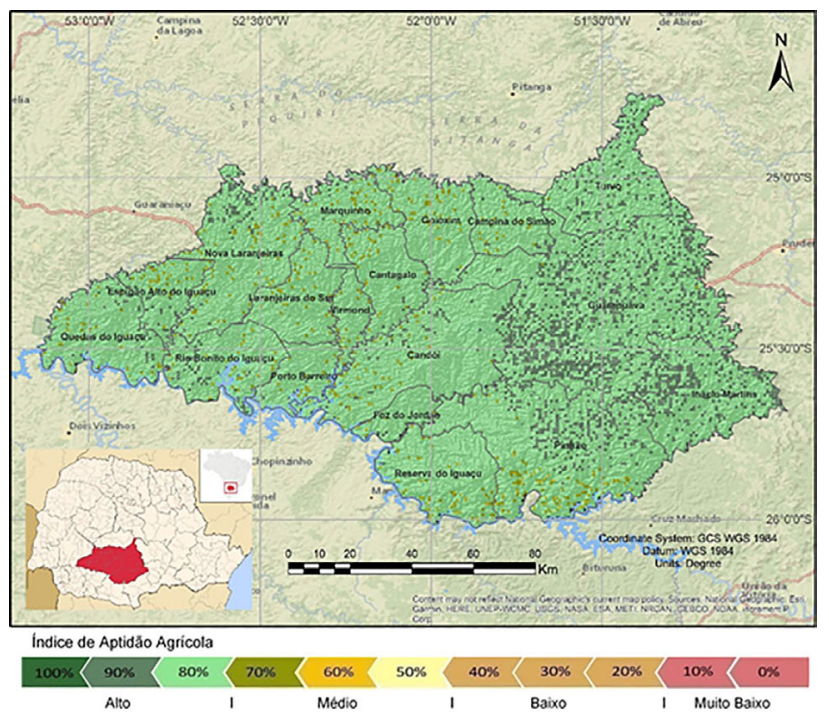

Figura 3 - Aptidão Agrícola para a cultura de trigo - Cenário base (19702000).
Tabela 1 - IAP - trigo, comparativo cenário base e cenários RCP4.5 e 8.5 (2040-2060).

\begin{tabular}{|c|c|c|c|c|c|c|c|}
\hline \multirow{3}{*}{ Trigo } & & & & \multicolumn{4}{|c|}{ Cenário (2040-2060) } \\
\hline & & \multicolumn{2}{|c|}{ Cenário (1970-2000) } & \multicolumn{2}{|c|}{ RPC4.5 } & \multicolumn{2}{|c|}{$\mathrm{RCP} 8.5$} \\
\hline & & Área (ha) & $\%$ & Área (ha) & $\%$ & Área (ha) & $\%$ \\
\hline \multirow{4}{*}{ IAP } & $60 \%$ & 0 & 0 & 42.014 & 3 & 171.389 & 12 \\
\hline & $70 \%$ & 42.986 & 3 & 892.222 & 63 & 1.213 .750 & 85 \\
\hline & $80 \%$ & 1.240 .139 & 87 & 489.097 & 34 & 38.194 & 3 \\
\hline & $90 \%$ & 140.208 & 10 & 0 & 0 & 0 & 0 \\
\hline
\end{tabular}

$90 \%$ foram totalmente substituídas por áreas que apresentam IAP de $80 \%$ e $70 \%$. No restante da área é possível observar uma redução de $52,77 \%$ do IAP de $80 \%$ e um aumento de $59,67 \%$ do IAP de $70 \%$, além do surgimento de áreas com $60 \%$ de IAP.

A projeção climática referente ao cenário $\mathrm{RCP} 8.5$ indica mudanças seguindo tendências de diminuição no IAP para o cultivo de trigo na maior parte da área de estudo. Expressivamente os municípios localizados a leste e extremo norte apresentaram redução de IAP $80 \%$ para $70 \%$. Com maior destaque para Guarapuava, Inácio Martins, Pinhão e Turvo em que áreas com IAP de $80 \%$ e $90 \%$ tiveram o índice reduzido para $70 \%$, sendo mantidas áreas com IAP $80 \%$ no extremo leste e mais na metade norte e nordeste da área.

Para a metade mais a oeste a estimativa indica que áreas com IAP 80 e 70\% apresentarão redução deste índice para aproximadamente $60 \%$. No restante observa-se uma redução de $84,45 \%$ em áreas com IAP de $80 \%$ e um aumento de $82,27 \%$ em áreas com IAP de 70\%, além do estabelecimento de 171.389 ha com $60 \%$ de IAP. Estas distribuídas nos municípios de Quedas do Iguaçu, Espigão

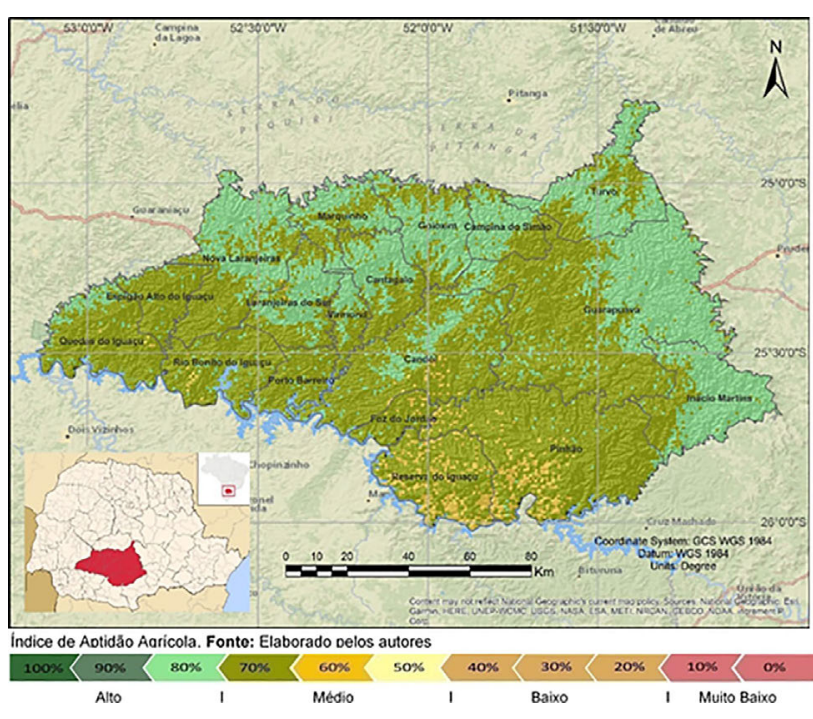

Figura 4 - Aptidão Agrícola para a cultura de trigo - Cenário futuro RCP4.5 (2040-2060). 


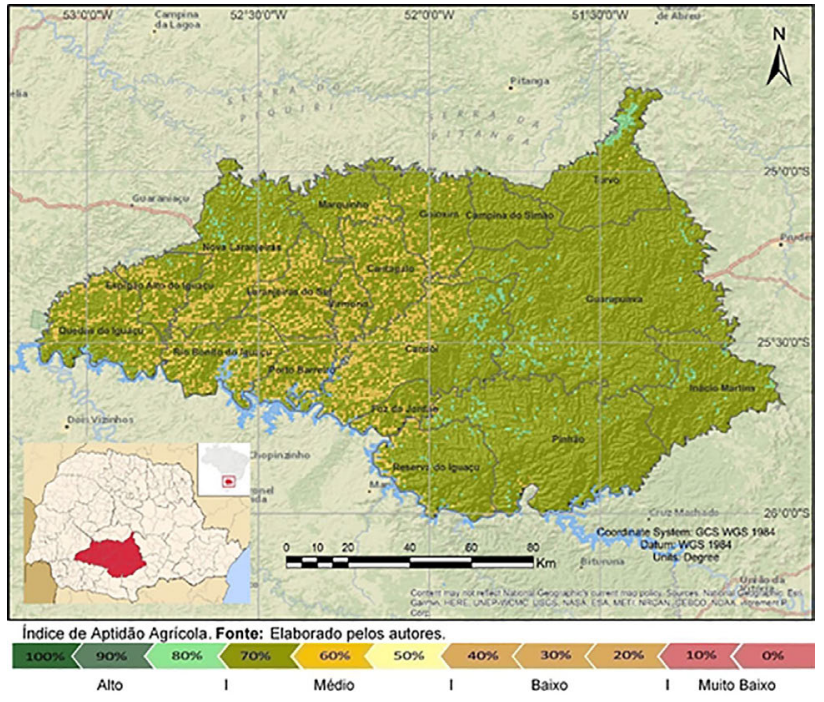

Figura 5 - Aptidão Agrícola para a cultura de trigo - Cenário futuro RCP8.5 (2040-2060).

Alto do Iguaçu, Nova Laranjeiras, Laranjeiras do Sul, Rio Bonito do Iguaçu, Porto Barreiro, Cantagalo Virmond, Foz do Jordão e Marquinho.

Conforme apresentado, para o ano de 2050 os cenários indicam estimativa de redução do índice de aptidão para o cultivo de trigo em praticamente toda a área de estudo, em comparação ao cenário base. Esta projeção de redução se deve diretamente ao aumento de temperatura. As diferenças mais distintamente evidentes entre os setores leste e oeste da área considerando o cenário RCP8.5 (Fig. 5), se devem também as características locais. Bassoi et al. (2016) ao descreverem as regiões tritícolas do Paraná, apresentam dados que classificam a parte leste da área como MRT1-(fria, úmida e alta) e a metade oeste como MRT2-(moderada, quente, úmida e alta/baixa). Estes dados são interessantes, pois, como pode ser verificado em IAPAR (2018), embora com diferenças climáticas sutis, a região tende a ser umas das mais frias do estado do Paraná, com clima Cfb, considerando a classificação climática de Köppen.

Os aproximados 38 mil ha remanescentes mantendo IAP de $80 \%$ no cenário RCP8.5, se apresentam distribuídos especialmente nos municípios a leste da área e pequena fração a extremo noroeste, com destaque para Nova Laranjeiras.

Na Fig. 6 verifica-se uma correlação linear negativa forte entre temperatura e o IAP para o trigo. O Índice relativo de temperatura, representa a variação proporcional média da temperatura na área de estudo, em porcentagem entre o observado no cenário base e o estimado para os cenários futuros.

Sobre o coeficiente $r$ de Pearson, este representa o ajuste linear da variação da distribuição da temperatura média observada e estimada na área de estudo. Observa-se

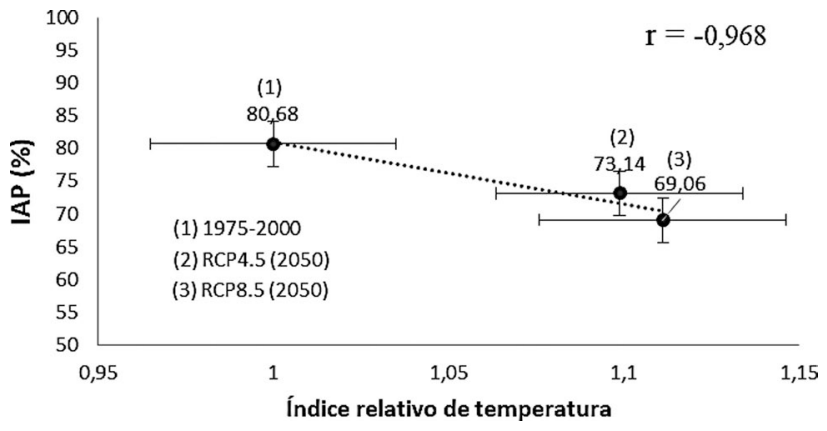

Figura. 6 - Correlação entre índice relativo de temperatura e Índice de aptidão agrícola para a cultura do trigo em diferentes períodos e cenários de projeção climática na microrregião de Guarapuava-PR.

que o índice de temperatura média mensal apresenta projeção de aumentar de 9,5 a $12 \%$ nos cenários RCP4.5 e 8.5 , respectivamente, resultando em elevação aproximada entre 1,4 a $2,0^{\circ} \mathrm{C}$ na média mensal entre abril a novembro, período do ciclo da cultura no campo. A relação inversa indica que este aumento do índice de temperatura média mensal está relacionado fortemente com a redução do IAP. $\mathrm{O}$ trigo é uma planta que se desenvolve melhor em regiões frias (BASSOI et al., 2016) sendo que o aumento de temperatura projetado para 2050 de acordo com ambos os cenários poderá contribuir potencialmente com a redução da aptidão agrícola das terras e, consequentemente, da produtividade das cultivares de trigo plantadas atualmente, conforme consideram Streck e Alberto (2006) e, de forma mais expressiva, para o cenário RCP 8.5 , em que o IAP é reduzido de $80 \%$ para $69 \%$.

A avaliação da relação entre o Índice relativo de precipitação e o índice de aptidão agrícola para o trigo é apresentada na Fig. 7, demonstrando correlação linear negativa moderada. Nesta figura, o Índice relativo de precipitação, representa a variação média da precipitação acumulada na área de estudo em proporção percentual entre o observado no cenário base e o estimado para os cenários futuros. Nota-se que no período do ciclo de cultivo do trigo, o índice relativo de precipitação apresenta projeção de aumentar entre aproximadamente $15 \%$ e $10 \%$ para os cen-

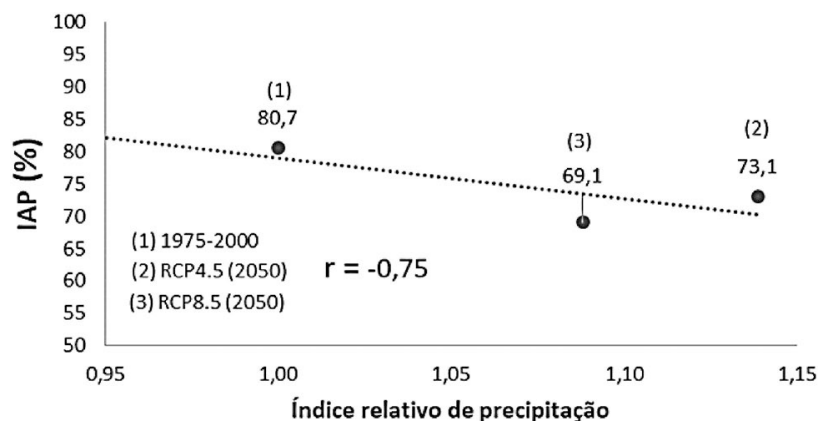

Figura 7 - Correlação entre Índice relativo de precipitação e Índice de aptidão agrícola para a cultura do trigo em diferentes períodos e cenários de projeção climática na microrregião de Guarapuava-PR. 
ários RCP4.5 e RCP8.5, em relação ao cenário base, contudo, o IAP tende a ser reduzido de $80,7 \%$ para $73 \%$ e $69 \%$, respectivamente.

Este resultado tende a ser controverso, considerando que o trigo responde positivamente ao aumento da disponibilidade hídrica durante o ciclo (Bassoi et al., 2016; Libardi e Costa, 1997), Contudo, a microrregião de Guarapuava apresenta elevados índices de precipitação, variando em média entre 90 e $200 \mathrm{~mm}$ mensais no período de abril a novembro (Wagner et al., 2009). E ainda as cultivares de trigo atualmente plantadas apresentam melhores produtividades em zonas mais frias do país (Silva et al., 2011; Santi et al., 2017).

O aumento de precipitação pluvial é fator agronômicamente benéfico para o trigo, e foi matemáticamente considerado na metodologia deste trabalho, conforme valoração 6,0 para precipitação, contra valor 4,0 para temperatura, na análise de múltiplos critérios aplicada (Fig.7), considerando Godoy e Assad (2002). Ainda assim, como os resultados apontam para tendências de reduções do IAP, entende-se que os efeitos positivos da projeção de aumento de precipitação sobre o IAP da área, tendem a ser anulados pela projeção de elevação da temperatura. Desta forma, é necessário maior atenção acerca da influência da variável temperatura em relação aos resultados apresentados.

Diferentes resutlados de pesquisas, como os apresentados por Streck e Alberto (2006) e por Pellegrino et al. (2007), apontam para ocorrência de aumento da temperatura e para consequencias negativas no desenvolvimento e produção de culturas agrícolas nas diferentes regiões brasileiras, e conforme Sposito e Faggian (2013) e Ebi et al. (2014) nos diferentes continentes. A partir disso, as perspectivas deste estudo não se restringem a microrregião de Guarapuava, pois estão associadas a tendencias climáticas que estão ocorrendo em áreas de cultivo de trigo em diferentes partes do mundo.

Para Dawson et al. (2015) e Santi et al. (2017) a elevação da temperatura é fator que deve ser considerado como crítico e tende a acarretar alterações na aptidão de áreas para cultivo de trigo e outros cereais de estação fria. A expectativa de aquecimento global com elevação da temperatura média no período de desenvolvimento do trigo, demonstra potencial de afetar o índice de aptidão das terras e, consequentemente, de reduzir o potencial produtivo cultura. Diante de perspectivas de aquecimento, Yang (2007) enfatiza a importância de programas de melhoramento que preconizam com maior ênfase as interações genótipo ambiente. Sendo assim, os resultados deste trabalho podem contribuir para programas de manejo e de melhoramento do trigo, visando os ajustes necessários e possíveis para o adequado desenvolvimento e produção da cultura nos próximos anos.

Os resultados deste trabalho, não se restringem somente a microrregião de Guarapuava, alvo do estudo.
Pesquisas demonstram que as alterações climáticas, destacadamente com aumento da temperatura e mudanças nos padrões de precipitação pluvial, estão ocorrendo nos diferentes continentes. Desta forma, estes resultados tornam-se disponíveis para compor base para programas de melhoramento vegetal, com vistas a buscar disponibilizar cultivares de trigo produtivas nas novas condições climáticas futuras. Bem como alertam para a necessidade de desenvolvimento da agricultura com técnicas de sustentabilidade ambiental, como alternativas para um adequado desenvolvimento regional nas próximas décadas.

\section{Conclusões}

O principal objetivo deste trabalho foi avaliar os possíveis impactos das mudanças climáticas na cultura de trigo, utilizando os cenários climáticos RCP4.5 e RCP8.5 (IPCC) para o cálculo do índice de aptidão agrícola da cultura na microrregião de Guarapuava-PR.

O índice médio de aptidão agrícola para a cultura do trigo na microrregião de Guarapuava tende a ser reduzido de $80 \%$ no cenário base (anos 1970-2000) para $73 \%$ e $69 \%$ no ano de 2050 a partir de projeções considerando os cenários RCP4,5 e RCP8.5, respectivamente. Tais reduções serão menos intensas nos municípios localizadas a norte e leste e mais intensas naqueles localizados a oeste da área.

O fator de maior impacto para a redução do índice de aptidão agrícola do trigo nos próximos 30 anos, é a projeção de aumento da temperatura média mensal em aproximadamente 1,5 a $2{ }^{\circ} \mathrm{C}$ durante o ciclo de desenvolvimento da cultura no campo.

A tendência de aumento da precipitação pluvial não apresenta índices de restrições à aptidão agrícola para o cultivo de trigo nos próximos 30 anos na microrregião de Guarapuava. Entretanto observa-se que as avaliações não são referentes a distribuição adequada da precipitação em momentos do ciclo da cultura nem a riscos de estiagens ocasionais em diferentes locais da área.

Pesquisas com estimativas de Índice de aptidão agrícola (IAP), são altamente promissoras, apresentando-se como uma ferramenta para auxilio de tomada de decisões no agricultura. Porém, como tratam-se de experimentos pioneiro, existe necessidade de sequência de novas investigações, com vistas a traduzir os impactos das variações no IAP sobre a produtividade da cultura do trigo, ou de outras culturas, dados atualmente ainda restritos.

\section{Agradecimentos}

Agradecimento em especial a CAPES - Coordenação de Aperfeiçoamento de Pessoal de Nível Superior por auxilio a partir do Programa Doutorado Sanduíche no Exterior, processo PDSE - 88881.133586/2016-01. 


\section{Referências}

ALLEY, R.B.; LEMKE P.; REN, J. A Report of Working Group I of the Intergovernmental Panel on Climate Change Summary for Policymakers Summary for Policymakers. IPCC Climate Change. Cambridge: Cambridge University Press, 2007.

BASSOI, M.C.; FOLONI, J.; SIMONETI, S.; SILVA, S.R. Indicações Filotécnicas para Cultivares de Trigo da Embrapa no Paraná. Londrina: Embrapa Soja, 2 ed., 2016.

BATHRELLOS, G.D.; GAKI-PAPANASTASSIOU, K. Assessment of rural community and agricultural development using geomorphological-geological factors and GIS in the Trikala prefecture (Central Greece). Stochastic Environmental Research and Risk Assessment, v. 27, n. 2, p. 573-588, 2013.

BATJES, N.H. A homogenized soil data file for global environmental research: A subset of FAO, ISRIC, and NRCS profiles (Version 1.0). Working Paper and Prepring 95/10b, International Soil Reference and Information Center. Wageningen: FAO, 1995.

BAXTER, R.; HASTINGS, N.; LAW, A. Implantação do modelo HADGEM2 do uk-met office no inpe: resultados preliminares. Animal Genetics, v. 39, n. 5, p. 561-563, 2008.

BENEZATH, A.D.A. et al. Anuário do Instituto de Geociências UFRJ Análise do Impacto da Mudança do Clima na Precipitação sobre as Bacias do Rio Paraná e do Rio Paraguai através da Regionalização de Projeções Climáticas Using Dynamical Downscaling of Climate Projections. Anuário do Instituto de Geociências - UFRJ. www.anuario.igeo. ufrj.br, v. 42, p. 695-707, 2019.

BURKE, M.; HSIANG, L. M.; MIGUEL, E. Global non-linear effect of temperature on economic production. Nature, v. 527, p. 235-239, 2015.

CGIAR-CSI. Consortium for Spatial Information. Disponível em http://www.cgiar-csi.org/, acesso em 10/02/2020.

CHAO, Q; FENG, A. Scientific basis of climate change and its response. Global Energy Interconnection, v. 1, n. 4, p. 420-427, 2018.

CHEN, M.; SHI, W.; XIE, P.; SILVA, V.B.S.; KOUSKY, R.; HIGGINS, R.W.; JANOWIAK, J.E. Assessing objective techniques for gauge-based analyses of global daily precipitation. Journal of Geophysical Research Atmospheres, v. 113, p. 1-13, 2008.

COLLINS, W.J.; BELLOUIN, N.; DOUTRIAUX-BOUCHE.; M.; GEDNEY, N.; HALLORAN, P.; HINTON, T.; HUGHES, J.; JONES, C.D.; JOSHI, M.; LIDDICOAT, S.; CUNHA, G.R.; CAIERÃO, E.; ROSA, A.C. Informações técnicas para Trigo e Triticale safra 2015. VIII Reunião da Comissão Brasileira de Pesquisa de Trigo e Triticale, p. 229, 2015.

DAWSON, I.K.; RUSSEL, J.; POWELL, W.; STEFFENSON, B.; THOMAS, W.T.B.; WAUGH, R. Barley: A translational model for adaptation to climate change. New Phytologist, v. 206, p. 913-931, 2015.

DUJMOVIC, J.; DE TRE, G.; DRAGICEVIC, S. Comparison of multicriteria methods for land-use suitability assessment. Proceedings of the Joint 2009 International Fuzzy Systems Association World Congress and 2009 European
Society of Fuzzy Logic and Technology Conference, p. 1404-1409, 2009.

EBI, K.; HALLEGATTE, S.; KRAM, T. A new scenario framework for climate change research: Background, process, and future directions. Climatic Change, v. 122, p. 387400, 2014.

EMBRAPA - Empresa Brasileira de Pesquisa Agropecuária. Brasil em Relevo - Embrapa Monitoramento por Satélite. Disponível em Embhttps://www.cnpm.embrapa.br/ projetos/relevo/download. Acesso em 26/09/2017.

FALLAHPOUR, F.; AMINGHAFOURI, A.; BEHBAHANI, A. G.; BANNAYAN, M. The environmental impact assessment of wheat and barley production by using life cycle assessment (LCA) methodology. Environment, Development and Sustainability, v. 14, n. 6, p. 979-992, 2012.

FARAG, A.A.; ABDRABBO, A.A.M.; EL SHARKAWI, H.M.; ABOU-HADID, A.F. Comparison between SERES and RCP scenarios in temperature and evapotranspiration under different climate zone. Journal of Environmental Science, Toxicology and Food Technology, v. 10, n. 11, p. 54-64, 2016.

FERNANDES, P.L.; ROCCA DA CUNHA, G.J.L.V. Trigo no Brasil. Bases para Produçao Competitiva e Sustentável. Brasília: EMBRAPA, 488 p., 2011.

FERRETTI, V.; POMARICO, S. Ecological land suitability analysis through spatial indicators: An application of the analytic network process technique and ordered weighted average approach. Ecological Indicators, v. 34, p. 507519, 2013.

GIORGI, F. Climate change prediction. Climatic Change, n. 73, p. 239-265, 2005.

GODOY, M.J.S.; LOPES-ASSAD, M.L. Aptidão agrícola de terras com estimativa de risco climático para a cultura do milho utilizando geoprocessamento. Revista Brasileira de Ciência do Solo, v. 26, n. 3, p. 685-694. 2002.

GUNDA, T.; BAZUI, J.T.; NAY, J.; YEUNG, K.L. Impact of seasonal forecast use on agricultural income in a system with varying crop costs and returns: an empirically-grounded simulation. Environmental Research Letters, v. 12, n. 3, p. 1-13, 2017.

HENGL T.; HEUVELINK, G.B GRAELER, B.; PEBESMA, E. et al. Spatio-temporal interpolation of daily temperatures for global land areas at $1 \mathrm{~km}$ resolution. Journal of Geophysical Research: Atmospheres, v. 19, n. 5, p. 22942313, 2014.

HIJMANS, R.J.; SUSAN E.C.; JUAN L.P.; PETER G.J.; ANDY $J$. Very high resolution interpolated climate surfaces for global land areas. International Journal of Climatology, v. 25, p. 1965-1978, 2005.

IAPAR - Instituto Agronõmico do Paraná. Cartas Climáticas do Paraná/Classificação Climática. Disponível em http:// www.iapar.br/modules/conteudo/conteudo.php?conteudo $=2533$, acesso em 21/11/2018.

IBGE - Instituto Brasileiro de Geografia Estatistica. Censo Agropecuário 2017. Disponível em https://censos.ibge. gov.br/agro/2017/resultados-censo-agro-2017.html/, acesso em 10/05/2020.

IBGE - Instituto Brasileiro de Geografia Estatistica. Sirgas2000: O referencial Geocêntrico do Brasil. Ponto de Referência, v. 1, n. 1, p. 26, 2006. 
IBGE - Instituto Brasileiro de Geografia Estatistica. Estatísticas do Registro Civil 2014, v. 41, p. 1-81, 2014.

LIBARDI, V.C.M.; COSTA, M.B. Consumo d'água da cultura do trigo (Triticum aestivum, L.). Revista FZVA, v. 4, n. 1, p. 16-23, 1997.

MALCZEWSKI, J. GIS-based land-use suitability analysis: A critical overview. Progress in Planning, v. 62, n. 1, p. 365, 2004.

MARTIN, G.M.; BELLOUIN, N.; COLLINS, W.J.; CULVERWELL, I.D.; HALLORAN, P.R. et al. The HadGEM2 family of met office unified model climate configurations. Geoscientific Model Development, v. 4, p. 723-757, 2011.

MICHEL, T.; SCUSE, S. World Agricultural Supply and Demand Estimates. Washington: United States Departament of Agriculture, 2018.

MOHANTY, M.; PROBERT, M.E.; SAMMI-REDDYC, B.K.; DALALAD, R.C.; MISHRAC, A.K.; SUBBA-RAOC, A.; SINGHC, M.; MENZIESA, N.W. Simulating soybeanwheat cropping system: APSIM model parameterization and validation. Agriculture, Ecosystems and Environment, v. 152, n. 51, p. 68-78, 2012.

MRAZOVA, J.; FAGGIAN, R.; SPOSITO, V. Sustainable development of catchment land - Use for multifunctional agro ecological landscapes under a changing climate. Anais The IAFOR International Conference on Sustainability, Energy and the Environment, Honolulu, 2017.

O'NEILL, B.C.; KRIEGLER, E.; RIAHI, K.; EBI, K.L.; HALLEGATTE, S.; CARTER, T.R.; MATHUR, R.A new scenario framework for climate change research: The concept of shared socioeconomic pathways. Climatic Change, v. 122, n. 3, p. 387-400, 2014.

PELLEGRINO, G.Q.; ASSAD, E.D.; MARIN, F.R. Mudanças climáticas globais e a agricultura no Brasil. Mudanças Climáticas, n. 8, p. 39-162, 2007.

ROMEIJN, H.; FAGGIAN, R.; SPOSITO, V.; MOORE, G. Agricultural land suitability uncertainty determination using weight sensitivity for climatic inputs in a current and future timeframe. In: Practical Responses to Climate Change: Proceedings of the Engineers Australia Conference 2014. Barton: Engineers Australia, p. 188-197, 2014.

SAATY, R.W. The analytic hierarchy process-what it is and how it is used. Mathematical Modelling, v. 9, n. 3, p. 161-176, 1987.

SANTI, A.; VICARI, M.B.; PANDOLFO, C.; DALMAGO, G.A.; MASSIGNAM, A.M.; PASINATO, A. Impacto de cenários futuros de clima no zoneamento agroclimático do trigo na região Sul do Brasil. Agrometeoros, v. 25, n. 2, p. 303-311, 2017.

SEAB - Secretaria da Agricultura e do Abastecimento. Paraná, 2018. Levantamento da Produção Agropecuária. Disponível em http://www.agricultura.pr.gov.br/deral/ProducaoAnual, acesso em 21/03/2019.

SILVA, R.R.; BENIN, G.; SILVA, G.O. et al. Adaptabilidade e estabilidade de cultivares de trigo em diferentes épocas de semeadura, no Paraná. Pesquisa agropecuária Brasileira, v. 46, n. 11, p. 1439-1447, 2011.

SPOSITO, V.; FAGGIAN, R. Expert systems modeling for assessing climate change impacts and adaptation in agricultural systems at regional level. Open Journal of Applied Sciences, v. 3, n. 6, p. 369-380, 2013.

STRECK, N.A.; ALBERTO, C.M. Estudo numérico do impacto da mudança climática sobre o rendimento de trigo, soja e milho. Pesquisa Agropecuária Brasileira, v. 41, n. 9, p. 1351-1359, 2006.

VAN VUUREN, D.P.; EDMONS, J.; KAINUMA, M. The representative concentration pathways: An overview. Climatic Change, v. 109, n. 5, p. 5-31, 2011.

WAGNER, M.V.; JADOSKI, S.O.; SAITO L.R.; SUCHORONCZEK A.; SCABENI, C. Comportamento de características climáticas para o manejo de culturas agrícolas em Guarapuava, centro-sul do estado do Paraná. Pesquisa Aplicada \& Agrotecnologia, v. 2, n. 3, p. 41-60, 2009.

WorldClim. Maps, Graphs, Tables, and Data of the Global Climate, disponível em http://www.worldclim.org/, acesso em 10/02/2020.

YANG, R.C. Mixed-model analysis of crossover genotype environment interactions. Crop Science, v. 47, p. 1051-1062, 2007.

TEIXEIRA, L.A.R.; JADOSKI, S.O.; FAGGIAN, R.; SPOSITO, V.; MRÁZOVÁ, J. Estimativa das alterações de temperatura no ciclo de cultivo de trigo e cevada para o ano de 2050 em Guarapuava, Paraná-Brasil. Pesquisa Aplicada \& Agrotecnologia, v. 11, n. 3, p. 41-49, 2018.

TRENBERTH, K.E. Changes in precipitation with climate change. Inter-Research CR., v. 47, n. 1-2, p. 123-138, 2011.

License information: This is an open-access article distributed under the terms of the Creative Commons Attribution License (type CC-BY), which permits unrestricted use, distribution and reproduction in any medium, provided the original article is properly cited. 